\title{
Adaptive potential of human biceps femoris muscle demonstrated by histochemical, immunohistochemical and mechanomygraphical methods
}

\author{
Raja Dahmane · Srdjan Djordjevič • \\ Vika Smerdu
}

Published online: 23 January 2007

(C) International Federation for Medical and Biological Engineering 2007

Erratum to: Med Bio Eng Comput 44(11):999-1006

DOI 10.1007/s11517-006-0114-5

Unfortunately, an error occurred in the Acknowledgement and also in Fig. 2. The correct version of the first sentence of Acknowledgement is given below.

This study was supported by grants from the Slovenian Olympic Committee and the TMG-BMC d.d.o.

The correct Fig. 2 is given below:

The online version of the original article can be found at http://dx.doi.org/10.1007/s11517-006-0114-5

R. Dahmane $(\bowtie) \cdot$ V. Smerdu Institute of Anatomy, Medical Faculty,

University of Ljubljana, Korytkova 2, Ljubljana, Slovenia

e-mail: raja.dahmane@guest.arnes.si

R. Dahmane

University College for Health Studies,

University of Ljubljana, Ljubljana, Slovenia

S. Djordjevič

Institute of Kinesiology, University of Primorska,

Koper, Slovenia

S. Djordjevič

Department of Biology, University of Ljubljana,

Ljubljana, Slovenia 
Fig. 2 Fibre types (1, 2a, $2 \times$ and $2 c$ ) in human biceps femoris muscle demonstrated according to the reaction for mATPase at different preincubations (a 4.3, b 4.6, c 9.4) and immunohistochemical staining with monoclonal antibodies specific to MyHC isoforms: d BA-D5 (MyHC-1), e SC-71 (MyHC-2a), f BF-35 (specific to $\mathrm{MyHC}-2 \mathrm{a}$ if the sections are pre-treated in microwave oven. Bar is $100 \mu$

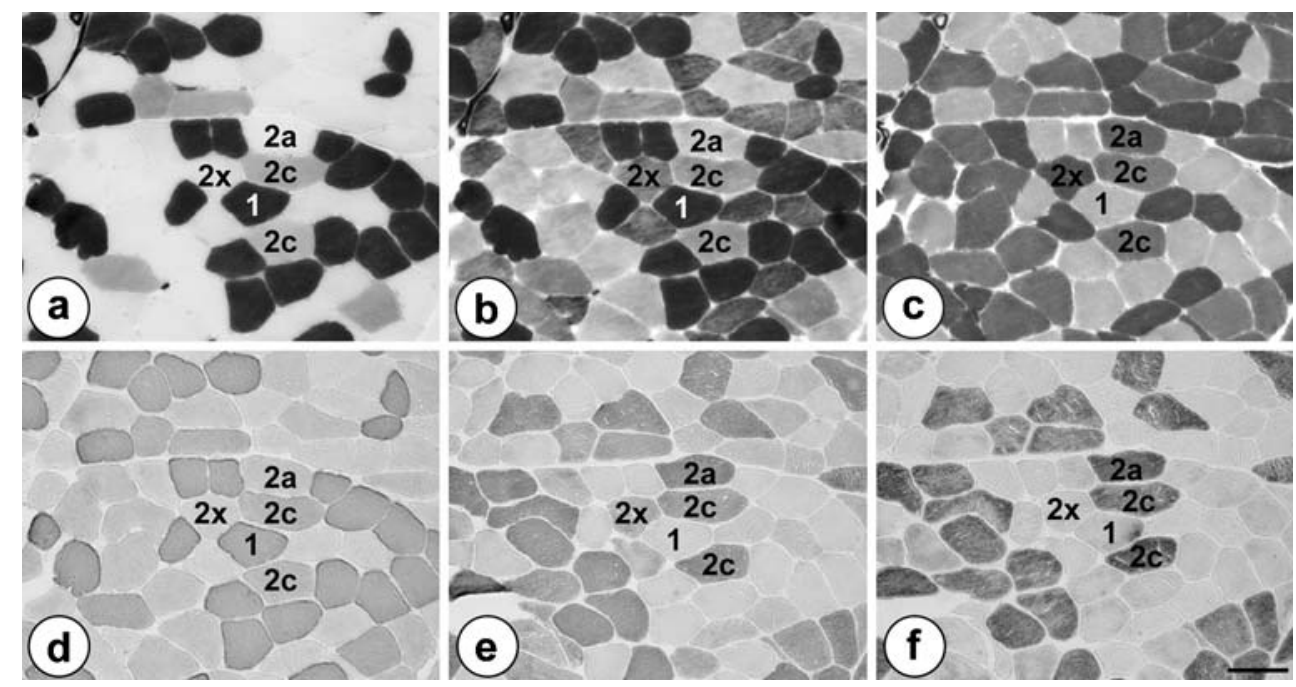

